\title{
On the electrostatic potential profile in biased molecular wires
}

Abraham Nitzan, Michael Galperin, Gert-Ludwig Ingold, and Hermann Grabert

Citation: The Journal of Chemical Physics 117, 10837 (2002); doi: 10.1063/1.1522406

View online: https://doi.org/10.1063/1.1522406

View Table of Contents: http://aip.scitation.org/toc/jcp/117/23

Published by the American Institute of Physics

\section{Articles you may be interested in}

The electrostatic potential profile along a biased molecular wire: A model quantum-mechanical calculation

The Journal of Chemical Physics 118, 3756 (2003); 10.1063/1.1539863

Molecular wire conductance: Electrostatic potential spatial profile

The Journal of Chemical Physics 112, 6834 (2000); 10.1063/1.481258

Generalized Formula for the Electric Tunnel Effect between Similar Electrodes Separated by a Thin Insulating Film

Journal of Applied Physics 34, 1793 (1963); 10.1063/1.1702682

Conductance spectra of molecular wires

The Journal of Chemical Physics 109, 2874 (1998); 10.1063/1.476841

Electron transfer at thermally heterogeneous molecule-metal interfaces

The Journal of Chemical Physics 146, 092305 (2017); 10.1063/1.4971293

Destructive quantum interference in electron transport: A reconciliation of the molecular orbital and the atomic orbital perspective

The Journal of Chemical Physics 146, 092308 (2017); 10.1063/1.4972572

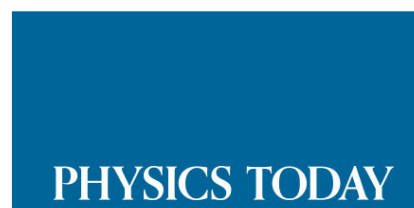

WHITEPAPERS
ADVANCED LIGHT CURE ADHESIVES

\section{READ NOW}

Take a closer look at what these environmentally friendly adhesive systems can do
PRESENTED BY

Q. MASTERBOND" 


\title{
On the electrostatic potential profile in biased molecular wires
}

\author{
Abraham Nitzan and Michael Galperin \\ School of Chemistry, The Sackler Faculty of Science, Tel Aviv University, Tel Aviv, 69978, Israel \\ Gert-Ludwig Ingold ${ }^{\text {a) }}$ \\ Institut für Physik, Universität Augsburg, Universitätsstrasse 1, D-86135 Augsburg, Germany \\ Hermann Grabert \\ Physikalisches Institut, Albert-Ludwigs-Universität, Hermann-Herder-Strasse 3, D-79104 Freiburg, Germany
}

(Received 5 August 2002; accepted 23 September 2002)

\begin{abstract}
The potential profile across a biased molecular junction is calculated within the framework of a simple Thomas-Fermi-type screening model. In particular, the relationship between this profile and the lateral molecular cross section is examined. We find that a transition from a linear potential profile to a potential that drops mainly near the molecule-metal contacts occurs with increasing cross-section width, in agreement with numerical quantum calculations. (C) 2002 American Institute of Physics. [DOI: 10.1063/1.1522406]
\end{abstract}

\section{INTRODUCTION}

Molecular wires are molecules that bridge between metallic leads to form a nano-conductor whose current-voltage characteristic reflects the electronic structure of the molecule. The conductance may be controlled via its dependence on molecular properties. Equally important is the use of such molecular junctions as capacitive elements in nanoelectronics.

Understanding the behavior of such junctions under potential bias is a prerequisite for elucidating their transport properties. The importance of this issue for the conductance of molecular junctions was recently emphasized by Datta and co-workers, ${ }^{1,2}$ who have shown, within a simple extended-Hückel (EH) model for $\alpha, \alpha^{\prime}$-xylyl dithiol bridging between two gold leads, that the potential profile (imposed on the molecule as input to the EH calculation) had a profound effect on quantitative as well as qualitative aspects of the calculated current-voltage characteristic. The best fit to experimental results was obtained from a model that assumed (a) a flat potential profile in the interior of the molecular bridge, i.e., the potential drop occurs only at the molecule-lead contacts and (b) a symmetric distribution of the potential drop at the two contacts, i.e., for a total voltage $\Phi$ the potential drops at each molecular edge by $\Phi / 2$.

This picture is supported by a recent model calculation by Mujica et al., ${ }^{3}$ where the Schrödinger equation (on the Hartree level) was solved in conjunction with the Poisson equation to yield both the electronic structure and the spatial distribution of the electrostatic potential. ${ }^{4}$ It was found that beyond a screening distance of the order of one to three atomic lengths the potential is flat along the model molecular chain.

$A b$ initio calculations with open system boundary conditions reveal a different picture: Lang and Avouris ${ }^{5}$ have found for a chain of seven carbon atoms connecting between

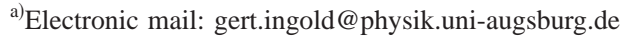

jellium leads that a substantial part of the voltage drop occurs along the carbon chain itself. Damle et al. ${ }^{6}$ have obtained similar results for a chain of gold atoms as well as for a molecular wire-phenyl-dithiol bridging between two gold electrodes. ${ }^{7}$ In an earlier work, Pernas et al. ${ }^{8}$ have determined that the potential along a model molecular wire is flat in the absence of scattering centers, however these authors have derived the local potential from a questionable local charge neutrality condition.

Recently, Weber et al. ${ }^{9}$ have considered the voltage profile across 9,10 - $B$ is $\left(\left(2^{\prime}\right.\right.$-para-mercaptophenyl)-ethinyl)anthracene coupled to two $\mathrm{Au}_{29}$ clusters. Their density functional theory calculations thus go beyond the assumption of a structureless metallic electrode and take into account the specific properties of the bond between the molecule and the gold atom in its local environment.

On the experimental side, Bachtold et al. ${ }^{10}$ have used scanned probe microscopy of both single-walled and multiwalled carbon nanotubes (SWNT and MWNT, respectively) to measure the potential drop along such nanotubes connecting between two gold electrodes. They find an approximately linear drop of the potential in a MWNT of diameter $9 \mathrm{~nm}$ while for a metallic SWNT bundle of diameter $2.5 \mathrm{~nm}$ the potential is flat beyond the screening regions at the tube edges. It should be emphasized that these experiments cannot be related directly to the above-discussed calculations. The nanotube length is a few microns and impurity and defect scattering may be effective as is most certainly the case in the MWNT measurement. The flat potential seen in the metallic SWNT measurement is in fact a remarkable observation implying a very long mean free path $(>1 \mu \mathrm{m})$ for electrons in these room temperature structures.

It is clear from the above-described studies that while the computational methodology for evaluating the potential distribution on a biased molecular wire is available, a complete understanding of the way this distribution behaves in different wires is lacking. In this respect simple models that focus on generic properties of conducting constrictions in- 


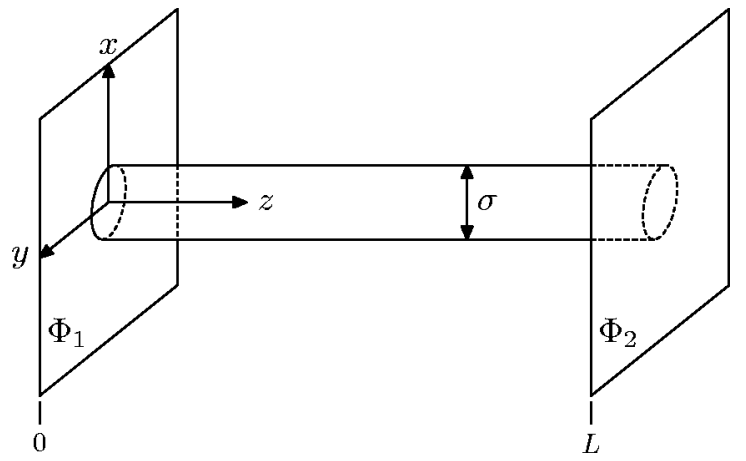

FIG. 1. The general setup contains a molecular wire modeled as a cylinder of length $L$ and diameter $\sigma$ between two perfect conductors with potentials $\Phi_{1}$ and $\Phi_{2}$.

cluding molecular wires are useful. The calculations of Pernas et al. ${ }^{8}$ provide such a model that is however hampered, as already stated by the restriction of local charge neutrality. The calculation of Mujica et al. ${ }^{3}$ is also based on a generic molecular model, however, by using a one-dimensional Poisson equation for the electrostatic potential these authors tacitly assume a molecular bridge whose lateral dimension is much larger than the screening length. In view of the fact that the width of molecular wires is often just a few angstroms, such an assumption is overly restrictive. Clearly, the magnitudes of the lateral width of the wire and the screening length should be important generic quantities for this issue. In this paper we present a simple model calculation that takes the relative magnitudes of these variables explicitly into account. We describe the model in Sec. II and present the calculation in Sec. III and some results and discussion in Sec. IV.

\section{THE MODEL}

The molecular wire is modeled as a cylinder of length $L$ and diameter of order $\sigma$ (the exact way in which $\sigma$ enters into the model calculation is explained in the following), perpendicular to and connecting between two planar metal electrode surfaces. As depicted in Fig. 1, the cylinder is oriented parallel to the $z$ axis, with its axis going through the origin in the $x y$ plane. The two electrodes are assumed to be ideal conductors implying a constant potential on the entire surface of each electrode. We set the potentials at the left and right wire-electrode interface to be $\Phi_{1}=\Delta / 2$ and $\Phi_{2}$ $=-\Delta / 2$, respectively. In view of Eq. (2), this guarantees a vanishing mean potential in the $z$ direction and thus a neutral molecule. Finally, we restrict the discussion of the potential profile to blocking junctions between electrodes and molecule so that no current is flowing.

As in Ref. 3 we assume that the wire material does have screening capacity, and is characterized by a screening length $\lambda$. It should be noted that the existence, nature, and size of such screening length in molecular systems is an open question with a probably nonunique answer. Molecules with large gaps between their highest occupied and lowest unoccupied molecular orbitals (HOMO and LUMO) will obviously screen poorly, while highly conjugated molecules with low HOMO-LUMO gap will screen relatively well.
In the present discussion we assume that over the relevant length scales (of order $L$ ) screening is described by a Poisson equation

$$
\nabla^{2} \Phi=-4 \pi \rho .
$$

According to the cylinder symmetry of the molecule, the charge density $\rho\left(r_{\|}, z\right)$ depends on the radial distance $r_{\|}$ from the wire axis and the position $z$ along the wire. In transversal direction, the charge density is assumed to be determined by a given molecular electron distribution represented by a factor $F\left(r_{\|}\right)$. The longitudinal part depends on the potential along the molecular axis. The screening is then described by

$$
4 \pi \rho\left(r_{\|}, z\right)=-\frac{1}{\lambda^{2}} F\left(r_{\|}\right) \Phi(0, z),
$$

which together with Eq. (1) will allow us to determine the potential profile.

Any assumption about the functional form of $F\left(r_{\|}\right)$is in fact an assumption about the confinement of the molecular charge distribution in the molecular cylinder and in our generic model it is sufficient to take a form that reflects the molecular thickness $\sigma$. Other details of $F\left(r_{\|}\right)$are expected to be of secondary importance.

In the three-dimensional Thomas-Fermi model for screening in a gas of electrons with charge $e$ and mass $m_{e}$, the screening length $\lambda$ of Eq. (2) is related to the electron density $n$ by

$$
\lambda=\left(\frac{E_{F}}{6 \pi n e^{2}}\right)^{1 / 2}
$$

with the Fermi energy

$$
E_{F}=\frac{\left(3 \pi^{2} n\right)^{2 / 3} \hbar^{2}}{2 m_{e}} .
$$

At metallic electron densities $\lambda$ is typically of the order of $1 \AA$. To have efficient screening in a molecular system electrons (or holes) must occupy molecular states that are effectively delocalized over the length of the bridge. Charge doping by transfer from the metal electrode to the molecular bridge may be one source of such electrons. Their density is expected to be considerably lower than metallic, implying a larger characteristic screening length. We expect that a calculation based on Eqs. (1) and (2) that uses metallic electron density to estimate $\lambda$ will provide an upper bound on the effective screening in a molecular wire.

\section{THE POTENTIAL DISTRIBUTION}

Using the model described in Sec. II, our problem is to solve the equation

$$
\nabla^{2} \Phi\left(r_{\|}, z\right)=\frac{1}{\lambda^{2}} F\left(r_{\|}\right) \Phi(0, z)
$$

in the range $0 \leqslant z \leqslant L$ subject to the boundary conditions

$$
\Phi\left(r_{\|}, 0\right)=\Delta / 2, \quad \Phi\left(r_{\|}, L\right)=-\Delta / 2 .
$$

It is convenient to decompose the full potential

$$
\Phi\left(r_{\|}, z\right)=\Phi_{0}(z)+\phi\left(r_{\|}, z\right)
$$


into a first term describing the bare potential

$$
\Phi_{0}(z)=\Delta\left(\frac{1}{2}-\frac{z}{L}\right)
$$

in the absence of a molecule and a second term which reflects the additional potential $\phi\left(r_{\|}, z\right)$ satisfying the boundary conditions $\phi\left(r_{\|}, 0\right)=\phi\left(r_{\|}, L\right)=0$.

The resulting differential equation

$$
\nabla^{2} \phi\left(r_{\|}, z\right)=\frac{1}{\lambda^{2}} F\left(r_{\|}\right)\left[\Phi_{0}(z)+\phi(0, z)\right]
$$

may be solved by the Fourier ansatz

$$
\phi\left(r_{\|}, z\right)=\int \frac{d^{2} k_{\|}}{(2 \pi)^{2}} e^{i \mathbf{k}_{\|} \cdot \mathbf{r}_{\|}} \sum_{n=1}^{\infty} \hat{\phi}_{n}\left(k_{\|}\right) \sin \left(\frac{\pi n}{L} z\right) .
$$

After expressing the bare potential profile in terms of a Fourier series one arrives at

$$
\begin{aligned}
\hat{\phi}_{n}\left(k_{\|}\right)= & -\frac{1}{\lambda^{2}\left[k_{\|}^{2}+(\pi n / L)^{2}\right]} \hat{F}\left(k_{\|}\right) \\
& \times\left[\Delta \frac{1+(-1)^{n}}{\pi n}+\int \frac{d^{2} k_{\|}^{\prime}}{(2 \pi)^{2}} \hat{\phi}_{n}\left(k_{\|}^{\prime}\right)\right],
\end{aligned}
$$

where

$$
\hat{F}\left(k_{\|}\right)=\int d^{2} r_{\|} e^{-\mathbf{i} \mathbf{k}_{\|} \cdot \mathbf{r}_{\|}} F\left(r_{\|}\right) .
$$

For the potential profile along the molecular axis, only the transversal integral over the Fourier coefficients $\hat{\phi}_{n}\left(k_{\|}\right)$is needed which may easily be obtained from Eq. (11). Due to the symmetry of the bare potential only even Fourier coefficients are found to contribute. We thus arrive at our main result describing the potential profile along the molecule

$$
\Phi(0, z)=\Phi_{0}(z)-\frac{\Delta}{\pi} \sum_{n=1}^{\infty} \frac{F_{n}}{n\left(1+F_{n}\right)} \sin \left(\frac{2 \pi n}{L} z\right) .
$$

The coefficients $F_{n}$ accounting for the influence of screening are given by

$$
\begin{aligned}
F_{n} & =\frac{1}{\lambda^{2}} \int \frac{d^{2} k_{\|}}{(2 \pi)^{2}} \frac{\hat{F}\left(k_{\|}\right)}{k_{\|}^{2}+(2 \pi n / L)^{2}} \\
& =\frac{1}{\lambda^{2}} \int_{0}^{\infty} d r_{\|} r_{\|} F\left(r_{\|}\right) K_{0}\left(\frac{2 \pi n}{L} r_{\|}\right),
\end{aligned}
$$

where $K_{0}$ denotes a modified Bessel function. In the limit of very small screening length, $\lambda \rightarrow 0$, it is possible to show by evaluating the sum in Eq. (13) that the potential along the wire vanishes and the entire voltage drop occurs at the interface with the electrodes.

For the following discussion, it is convenient to introduce a measure of the deviation of the voltage profile $\Phi(0, z)$ from the linear behavior (8). Since the integral over $\Phi(0, z)$ $-\Phi_{0}(z)$ vanishes for a neutral molecule, we use instead

$$
\delta=\left[\frac{12}{\Delta^{2} L} \int_{0}^{L} d z\left(\Phi(0, z)-\Phi_{0}(z)\right)^{2}\right]^{1 / 2} .
$$

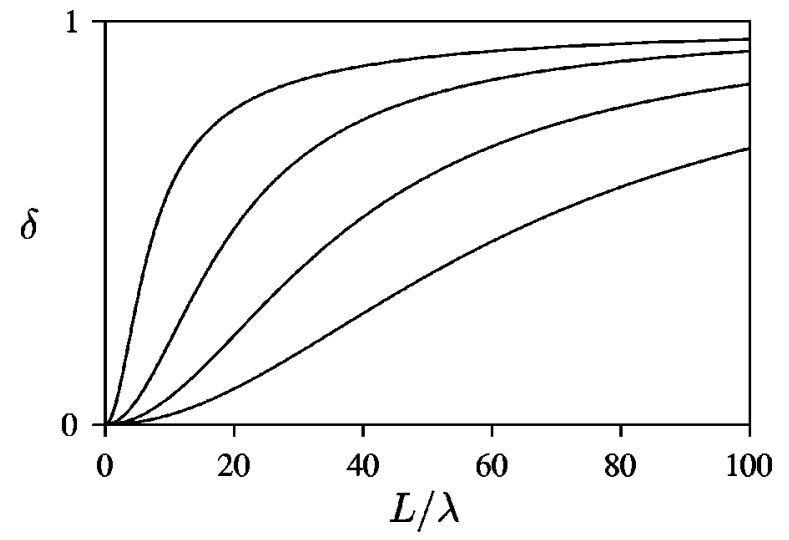

FIG. 2. The deviation $\delta$ [cf. Eq. (15)] of the voltage profile from the linear behavior is shown as a function of the ratio of wire length $L$ and screening length $\lambda$. The four curves correspond to $\sigma \rightarrow \infty$ [cf. Eq. (20)], $\sigma / L=0.05$, 0.02 , and 0.01 from the upper to the lower curve.

This quantity is normalized such that it equals 1 if the voltage drop occurs entirely at the ends of the molecule while it vanishes for a linear potential profile. Employing Eq. (13), one may express $\delta$ in terms of the coefficients defined by Eq. (14) as

$$
\delta=\frac{6^{1 / 2}}{\pi}\left[\sum_{n=1}^{\infty} \frac{F_{n}^{2}}{n^{2}\left(1+F_{n}\right)^{2}}\right]^{1 / 2} .
$$

\section{RESULTS AND DISCUSSION}

We now address the dependence of the potential profile on the width of the molecular wire and start with the limiting case of an infinitely thick molecule or, equivalently, a large number of molecules in parallel present between the two electrodes. Then, $F\left(r_{\|}\right)=1$ and one finds from Eq. (14)

$$
F_{n}=\left(\frac{L}{2 \pi \lambda n}\right)^{2} \text {. }
$$

Using

$$
\sum_{n=1}^{\infty} \frac{\sin (n x)}{n\left(n^{2}+a^{2}\right)}=\frac{\pi}{2 a^{2}} \frac{\sinh (a(x-\pi))}{\sinh (a \pi)}-\frac{x-\pi}{2 a^{2}} .
$$

Eq. (13) yields for the potential profile

$$
\Phi(0, z)=\frac{\Delta}{2} \frac{\sinh \left(\frac{L-2 z}{2 \lambda}\right)}{\sinh (L / 2 \lambda)} .
$$

The deviation from the linear voltage drop can be quantified by inserting Eq. (17) into Eq. (16). Evaluating the sum, one finds

$$
\delta=\left[1+24 \frac{\lambda^{2}}{L^{2}}-9 \frac{\lambda}{L} \operatorname{coth}\left(\frac{L}{2 \lambda}\right)-\frac{3}{2} \frac{1}{\sinh (L / 2 \lambda)}\right]^{1 / 2} .
$$

This result is shown in Fig. 2 as uppermost curve. In the limit of very large screening length, $\lambda \rightarrow \infty, \delta$ vanishes, thereby indicating the expected linear voltage drop. On the other hand, for very short screening length, $\lambda \rightarrow 0, \delta$ approaches one and the entire voltage drops at the interfaces between wire and electrode. 


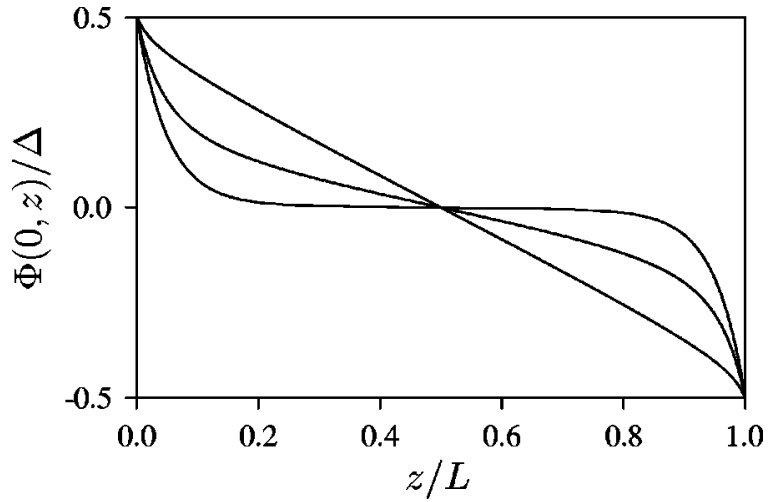

FIG. 3. The potential profile along a molecular wire computed from Eqs. (13) and (22) is shown for a screening length $\lambda / L=0.05$. The thickness parameter $\sigma / L$ takes the values $0.0125,0.05$, and 0.5 from the almost linear behavior to the voltage profile containing almost a plateau.

For the case of finite width, we employ the Gaussian charge distribution

$$
F\left(r_{\|}\right)=\exp \left(-r_{\|}^{2} / 2 \sigma^{2}\right) .
$$

This function is not normalized and therefore describes a charge distribution with a density in the center independent of the width $\sigma$. Such a situation arises when the diameter of a molecular layer can be controlled. Then, the charge density in the center appears in the screening length $\lambda$. In contrast, in the somewhat unrealistic case where the charge density on the wire is changed, the function $F\left(r_{\|}\right)$would have to be normalized.

One advantage of the Gaussian distribution (21) is the fact that the coefficients (14) may still be expressed analytically in terms of an exponential integral

$$
F_{n}=\frac{1}{2}\left(\frac{\sigma}{\lambda}\right)^{2} e^{\chi} \int_{\chi}^{\infty} d u \frac{e^{-u}}{u}
$$

with

$$
\chi=\frac{1}{2}\left(2 \pi n \frac{\sigma}{L}\right)^{2} .
$$

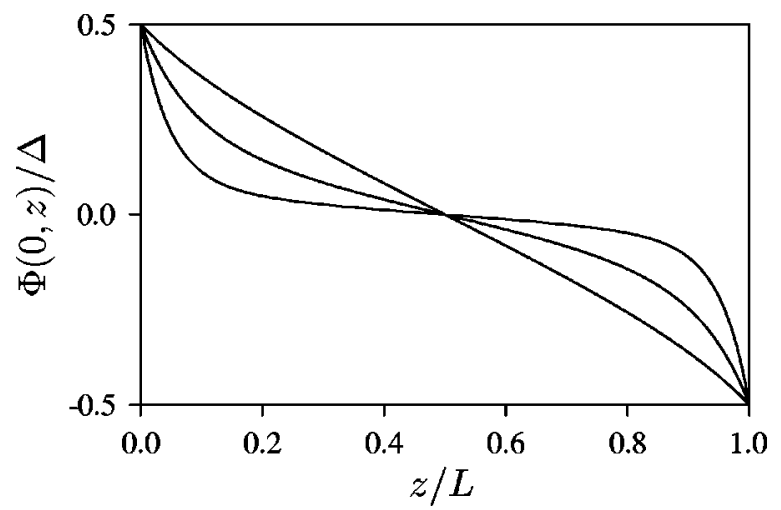

FIG. 4. Same as Fig. 3 but for fixed thickness parameter $\sigma / L=0.125$ and varying screening length $\lambda / L=0.25,0.1$, and 0.05 from the almost linear behavior to the voltage profile containing almost a plateau.

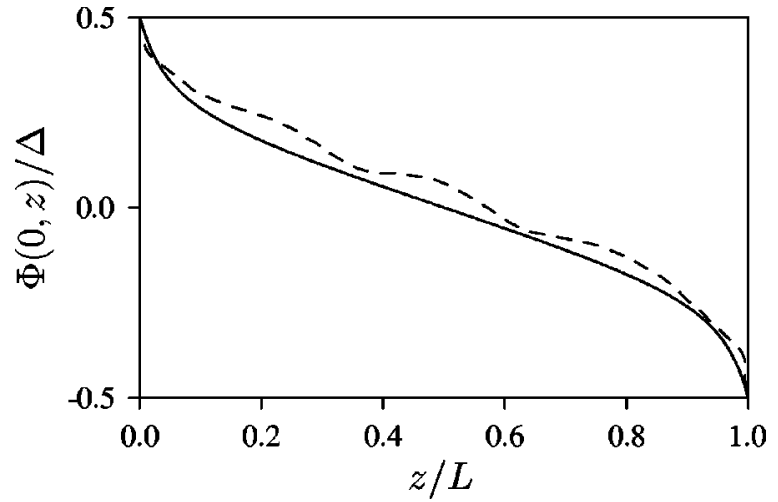

FIG. 5. The potential drop across a chain of six gold atoms placed between two gold electrodes as obtained from an ab initio calculation in Ref. 6 (dashed line) is fitted by a voltage profile (13) depicted as full line. The best fit was obtained for $\sigma / L=0.032$ and $\lambda / L=0.052$.

With this result the potential profile can be evaluated numerically according to Eq. (13) while the deviation from the linear voltage drop is obtained from Eq. (16).

In Fig. 2, the deviation $\delta$ of the voltage profile from the linear behavior (8) is shown for different values of the wire thickness $\sigma$. The uppermost curve corresponds to the limit of a thick molecular layer $\sigma \rightarrow \infty$, which was discussed earlier. The three other curves correspond to $\sigma / L=0.05,0.02$, and 0.01 from top to bottom. As these results demonstrate, a reduction of $\sigma$ causes a reduction of $\delta$ indicating that the voltage profile approaches the linear voltage drop. This behavior can be understood in terms of a reduction of screening due to the reduced molecular layer. However, this effect becomes only relevant for $\sigma \ll L$. As discussed earlier, the limit $\lambda \rightarrow 0$ leads to a constant potential along the molecular wire. Therefore, all curves shown in Fig. 2 tend to $\delta=1$ in this limit even though this is not shown in the figure.

We now turn to a discussion of the voltage profiles themselves. Figure 3 depicts results obtained from Eq. (13) using $F_{n}$ from Eq. (22) and $\Phi_{0}(z)$ as defined in Eq. (8). The dimensionless screening length $\lambda / L=0.05$ implies for a typical metallic screening length $\lambda=2$ a.u. a wire length of $L$ $=20$ a.u. The thickness parameter $\sigma / L$ for the three different curves are $0.0125,0.05$, and 0.5 , where the voltage profile becomes more and more linear as $\sigma$ decreases. As already mentioned, this may be understood in terms of the reduced screening. Figure 4 shows similar results for a wire with a ratio between the typical diameter and the wire length of $\sigma / L=0.125$. Here, the dimensionless screening length takes the decreasing values $\lambda / L=0.25,0.1$, and 0.05 with increasing deviation from the linear voltage profile.

In Fig. 5 we present a fit of our voltage profile to the $a b$ initio calculation of Ref. 6. A least square fit resulted in a screening length of $\lambda / L=0.052$ and a wire width of $\sigma / L$ $=0.032$. For a distance $L$ of approximately 34 a.u., this yields the reasonable value of 1.1 a.u. for the radial extent of the electron density.

The parameters $\sigma / L$ and $\lambda / L$ employed allow for a rather good approximation of the results of the ab initio calculation. However, there are two significant differences. The Friedel oscillations found in the quantum calculation cannot 
be obtained within our classical approach. In a tight-binding model description of the molecular wire, Friedel oscillations naturally arise from a breaking of electron-hole symmetry. ${ }^{11}$ Second, the systematic shift between the two voltage profiles in Fig. 5 indicates that the wire in the $a b$ initio calculation was charged while our wire is always assumed to be neutral.

It is obvious from these results that the ratio of the wire thickness to the screening length does constitute an important generic attribute that determines the general behavior of the potential bias distribution along a molecular wire. The relatively good fit obtained between the model calculations and the ab initio results for a chain of gold atoms using reasonable geometric parameters supports this conclusion. We note in passing that the flat potential distribution observed $^{10}$ for metallic single walled carbon nanotubes of thickness $\sim 20$ a.u. is consistent with the results shown in Fig. 3. One should keep in mind however that apart from its intrinsic simplicity, the model used in this work suffers from two important shortcomings. First, the use of a simple screening property as described by Eqs. (1) and (2) cannot be justified for all molecules, and certainly not for arbitrary distances. Even when such screening applies, the magnitude of the screening parameter $\lambda$ is not known and is expected to depend on the amount of equilibrium charge transfer between the wire and the metal leads. Second, a complete calculation of the potential profile along a molecular junction should take into account the fact that some of this drop may take place on the metal leads near the junction. Such a behavior was found in the calculation of Ref. 5.

\section{CONCLUSIONS}

The potential distribution along molecular wires in biased molecular junctions is determined in principle by the detailed electronic structure of the wire and by the response of this structure to the molecule-lead contacts and to the bias. The present study has identified the wire thickness as one of two generic attributes that largely determine the way the potential drops along the wire. Increasing this parameter leads to a crossover from a three-dimensional electrostatic problem to an effectively one-dimensional situation. The accompanying increase in screening causes a transition from a linear potential profile to a situation where the potential drops mostly at the interfaces between wire and electrode. The other, less accessible molecular property is its ability to screen a local charge. In the present model calculation we have used a simple screening length parameter to model this molecular property, but further studies are needed for a better characterization of this property.

\section{ACKNOWLEDGMENTS}

G.L.I. is grateful to S. Yaliraki and J. Lehmann for stimulating discussions. Three of us (A.N., G.L.I., and H.G.) would like to thank the Institute for Theoretical Physics at UCSB for hospitality during the workshop on "Nanoscience" where this work was started. This research was supported in part by the National Science Foundation under Grant No. PHY99-07949, by the Volkswagen-Stiftung under Grant No. I/77217 by the Israel Science Foundation, and by the Israel Ministry of Science.

${ }^{1}$ S. Datta, W. Tian, S. Hong, R. Reifenberger, J. I. Henderson, and C. P. Kubiak, Phys. Rev. Lett. 79, 2530 (1997).

${ }^{2}$ W. Tian, S. Datta, S. Hong, R. Reifenberger, J. I. Henderson, and C. P. Kubiak, J. Chem. Phys. 109, 2874 (1998).

${ }^{3}$ V. Mujica, A. E. Roitberg, and M. Ratner, J. Chem. Phys. 112, 6834 (2000).

${ }^{4}$ A similar methodology was applied in a different context by K. Hirose and M. Tsukada, Phys. Rev. B 51, 5278 (1995).

${ }^{5}$ N. D. Lang and P. Avouris, Phys. Rev. Lett. 84, 358 (2000).

${ }^{6}$ P. S. Damle, A. W. Ghosh, and S. Datta, Phys. Rev. B 64, 201403 (2001).

${ }^{7}$ In these $a b$ initio calculations the potential is calculated from the combination of the external field and the Hartree potential due to all molecular charges, using an extended molecular model that includes two cluster segments of the metal leads.

${ }^{8}$ P. L. Pernas, A. Martin-Rodero, and F. Flores, Phys. Rev. B 41, 8553 (1990).

${ }^{9}$ H. B. Weber, J. Reichert, F. Weigend, R. Ochs, D. Beckmann, M. Mayor, R. Ahlrichs, and H. v. Löhneysen, Chem. Phys. 281, 113 (2002).

${ }^{10}$ A. Bachtold, M. S. Fuhrer, S. Plyasunov, M. Forero, E. H. Anderson, A. Zettl, and P. L. McEuen, Phys. Rev. Lett. 84, 6082 (2000).

${ }^{11}$ S. Pleutin, H. Grabert, G.-L. Ingold, and A. Nitzan, this paper is now generally available on the preprint server, arXiv:physics/0209091. 